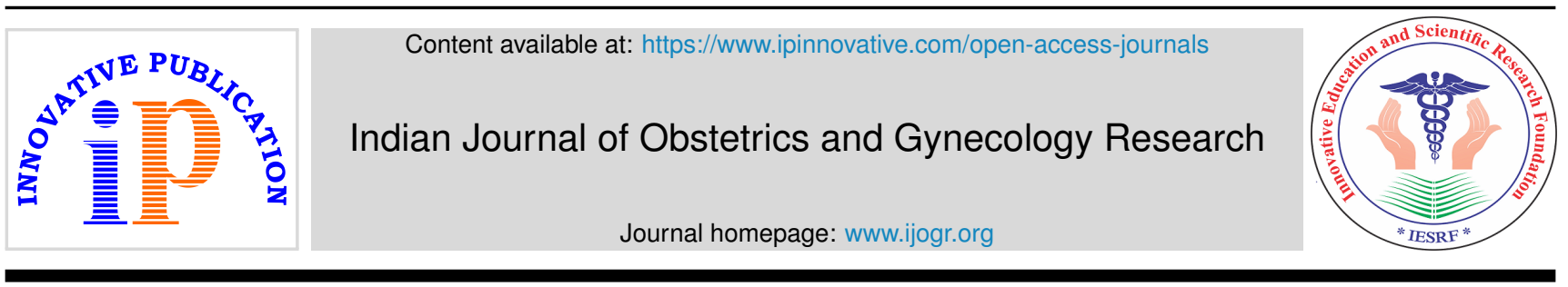

Original Research Article

\title{
Evaluation between low dose and high dose oxytocin for labour augmentation in fetal outcome
}

\author{
Girija C Bellad ${ }^{1}$, Mahesweta Guru ${ }^{1}$ * \\ ${ }^{1}$ Dept. of Obstetrics \& Gynaecology, Gujarat Adani Institute of Medical Science, Kutch, Gujarat, India
}

\section{A R T I C L E I N F O}

\section{Article history:}

Received 30-06-2020

Accepted 04-08-2020

Available online 07-12-2020

\section{Keywords:}

Oxytocin

Augmentation

High dose

Low dose

Functional dystocia

Labour duration

Maternal and fetal outcome

\begin{abstract}
A B S T R A C T
Aim: In the current study we intend to measure the effectiveness and protection of high and low dose oxytocin for rise of labour, on process of delivery.

Materials and Methods: One hundred pregnant women needed growth of labor forinadequate uterine contractions, even 1 hour after ARM [If membranes intact], and cervical dilatation is at least $3 \mathrm{~cm}$ or more. These cases were selectedrandomly and were assigned to either a low dose $(2.5 \mathrm{mU} / \mathrm{min})$ or a high dose $(5$ $\mathrm{mU} / \mathrm{min}$ ) regimen. Study included equal number of primigravida and multigravida in each group.

Results: High dose oxytocin group was associated with significant shorter duration of labor, as indicated by shortened augmentation to full dilatation and augmentation to delivery gap in primigravidae contrast to low dose group, but not in multigravdia. Both in multigravida and primigravdia maximum oxytocin dose was high with high dose regimen compared to low dose.

Conclusion: High dose oxytocin is better to low dose oxytocin for labour augmentation for efficient dystocia in primigravdia. High dose oxytocin augmentation in primigravdia is connected with considerable decrease in first stage of labour without any unpleasant perinatal and maternal morbidity or mortality.
\end{abstract}

(C) This is an open access article distributed under the terms of the Creative Commons Attribution License (https://creativecommons.org/licenses/by/4.0/) which permits unrestricted use, distribution, and reproduction in any medium, provided the original author and source are credited.

\section{Introduction}

Labor dystocia (LD) is a usually recognized dilemma in recent delivery care with three described probable causes: incompetent uterine action, occipital posterior position and cephalopelvic imbalance. incompetent uterine action is the mainly widespread problem of labor in primiparous women. ${ }^{1}$ Labour duration has shown a wide variation in different women, and slow labour progress is frequent in nulliparous women. It is connected with childbirth difficulties, distress for fetal comfort, and harmful birth practices, and is leading sign for spontaneous caesarean section in labour. ${ }^{2,3}$

Complex labour is considered by unusually sluggish labour development take place from incompetent uterine contractions, uncharacteristic fetal appearance or place, derisory bony pelvis or abnormalities of the pelvic soft

\footnotetext{
* Corresponding author.

E-mail address: shwetaguru412@gmail.com (M. Guru).
}

tissues of the mother. Data suggest that up to one third of first-time mothers practice hold-up in the first phase of labour. $^{4}$

Augmentation of labour is the procedure of stimulating the uterus to raise the occurrence, period and strength of contractions after the onset of impulsive labour. ${ }^{5}$ It has frequently been used to delight deferred labour when uterine contractions are measured to be inadequately tough or unsuitably synchronized to dilate the cervix. Labour augmentation has conventionally been carry out with the utilize of intravenous oxytocin infusion and/or artificial rupture of amniotic membranes. ${ }^{6}$ The process aims to curtail labour in order to avert difficulties relating to undue continuance, and to avoid caesarean section. There is proof that a major percentage of women with simple pregnancies are subjected to usual augmentation of labour with oxytocin. ${ }^{7,8}$

Oxytocin for the rationale of augmentation and induction of labor is most commonly used medications in obstetrics. 
Modern researches explians that oxytocin is used in over $50 \%$ of laboring women in various hospitals. ${ }^{9}$ Yet, there is tremendous variability in the dose and dosing interval in clinical practice. Oxytocin is the most frequently medication used for labor induction in obstetrics. ${ }^{10}$ Oxytocin is frequently used in contemporary obstetric practice to augment uterine movement, in cases in which the labor process has failed, with the aim to enable it to progress to a vaginal delivery. ${ }^{11}$

The utilization of oxytocin indicated for the management of labor dystocia, since it can decrease the rates of caesarean sections. ${ }^{12}$ Dystocia has been explaining as one of the major sign for caesarean section, in circumstances in which there is cessation in the procedure that would effect in a normal and artless delivery. Present study was done with an aim to measure the efficacy and protection of high and low dose oxytocin for augmentation of labour, on method of delivery.

\section{Materials and Methods}

The present study included the 50 singleton term pregnant women who were admitted in the hospital. The study was approved from the institution review board and the ethical clearance certificate was obtained from the committee. The patients were informed about the study and the informed consent was signed by them. These cases were selected randomly and were assigned to either a low dose or a high dose regimen. Study included equal number of primigravida and multigravida in each group. The inclusion criteria were as followed Primi/multigravida, singleton pregnancy, cephalic presentation, no medical complication and requiring augmentation of labor. The exclusion criteria were as follows multiple pregnancy, mal presentation, history of caesarean section and meconium stained liquor.

The clinical and basic investigations were done as soon as the patient was admitted in the labor room. The general condition, BP, temperature, CVS assessed, examination of abdomen, uterine contraction, vaginal examination, and pelvis assessments were done.

When patient enters active phase of and if membranes was intact amniotomy was performed. After 1 hour if uterine contraction are inadequate $[<4$ contraction in $10 \mathrm{~min}]$ or there was no cervical changes for 1 hour after ARM, oxytocin augmentation was used. Oxytocin augmentation was used when uterine contractions are inadequate and when cervical dilatation is at least $3 \mathrm{~cm}$ in case of PROM

Cervical examination was performed every two hours after starting augmentation and whenever clinically indicated and assessment of cervical effacement, dilation, membrane status, station of head done.

The state of membranes "I" if membranes were intact, ' $\mathrm{C}$ ' if membranes were ruptured and liquor clear. Moulding of head at initial examination and subsequent vaginal examination was noted. The most important measures of progress in labour, the rate of dilatation of cervix and the rate of descent of the fetal presenting part, are recorded by plotting the cervical dilatation on the vertical line on the left hand side of the graph in centimetres from 0 to 10 against the elapsed time which is plotted on the horizontal line in hours.

The uterine contractions were plotted on the graph below the cervicograph. Uterine activity is assessed by abdominal palpation. The examining hand is placed between the umbilicus and uterine fundus. The frequencies, duration of uterine contractions are quantified over 10 minutes period contractions are assessed at interval of 30 minutes. The frequency was assessed by counting the number of contractions occurring during 10 minutes period was measured in seconds and the number of blocks representing frequency were filled in by dots if the duration was less than 20 seconds, cross hatched if less than 40 seconds and blocked out if more than 40 seconds.

Infusion started at rate of $10 \mathrm{drop} / \mathrm{min}[6.2 \mathrm{mU} / \mathrm{min}$ or $3.2 \mathrm{mU} / \mathrm{min}]$. If adequate contractions was not achieved by the end of half hour, infusion rate increased by another 10 drop min, until adequate contractions obtained avoiding hyper stimulation. Below this uterine contraction on the graph, mother's temperature, pulses, BP were recorded. Urine examination for glucose, albumin and acetone were done and recorded in the graph. Note on augmentation delivery interval, duration of 2 nd stage mode of delivery, were made.

Statistical analysis was performed with student 't' test and analysis of variance for continuous data. Chi-square test for categorical data and group averages were reported as mean \pm standard deviation. A 'P' value of $<0.05$ was considered statistically significant.

\section{Results}

Total 50 patients were incorporated in the study. Equivalent number of primigravia and multigravida were disseminated in the study group. The groups were also compatible in respect to their cervical dilatation. When the age, weight and gestational age was evaluated amid the two dosage groups, no statistical significance difference was found.

When high dose oxytocin used for labor augmentation, it was associated with a significant shortening of labor duration as demonstrated by shortened augmentation to delivery interval (AD; high dose: $269 \pm 29.3 \mathrm{~min}$; low dose : $285 \pm 41.2 \mathrm{~min} ; \mathrm{p}=0.006)$, irrespective of parity. Time from augmentation to full dilation was also significantly shorter in high dose group. From Table 1 it can be concluded that high dose oxytocin regimen associated with shorter labor. Even though labor duration was abridged in high dose group, there was no dissimilarity in period of second stage and third stage of labor.

High dose augmentation group had important limitation of augmentation to complete dilation interval and augmentation to delivery gap, when evaluated in two 
Table 1: Different labour duration parameters in the study

\begin{tabular}{lcccc}
\hline Characteristics & High dose & Low dose & t & P value \\
AD & $305.2 \pm 35.2$ & $225.3 \pm 49.2$ & 2.21 & 0.001 \\
AFD & $269 \pm 29.3$ & $285 \pm 41.2$ & 2.16 & 0.006 \\
$2^{\text {nd }}$ stage & $33.2 \pm 8.1$ & $35.3 \pm 9.3$ & 0.51 & 0.23 \\
$3^{\text {rd }}$ stage & $4.9 \pm 0.9$ & $4.2 \pm 1.0$ & 1.02 & 0.25 \\
\hline
\end{tabular}

$\mathrm{AFD}=$ Augmentation to full dilatation interval

$\mathrm{AD}=$ Augmentation to delivery interval

Table 2: Maximum dose of oxytocin inprimi and multigravdia

\begin{tabular}{lccc}
\hline Maximum oxytocin dose & High Mean \pm SD & Low Mean \pm SD & Significance \\
Prmi + Multi & $12.2 \pm 5.1$ & $9.4 \pm 4.2$ & $<0.001$ \\
Primi & $17.2 \pm 4.5$ & $9.2 \pm 3.2$ & $<0.001$ \\
Multiple & $12.9 \pm 6.2$ & $12.4 \pm 3.4$ & 0.15 \\
\hline
\end{tabular}

oxytocin groups in primi gravidae. Slightly significant restriction of second stage labor was renowned in high dose regimen, with no dissimilarity in period of third stage labor.

Although, high dose oxytocin regimen was connected with significant reduction in labor duration (shortening of AFD, $A D$ ) in primigravida, the difference was not significant when two dosing regimen is compared in multigravdiae. There was no dissimilarity in the duration of labor, AFD, AD, 2nd stage of labour with 3rd stage of labor when high and low dose regimens are contrast to multigravidae.

The maximum oxytocin dose was significantly high in high dose group. When compared according to parity, the significant difference was noted only in primigravdia, but no difference in multigravdia. In primigravida, maximum oxytocin dose was significantly high in high dose group (high dose : $15.2 \pm 3.2 \mathrm{mU} / \mathrm{min}$; low dose : $9.2 \pm 3.0$ $\mathrm{mu} / \mathrm{min} ; \mathrm{p}<0.001$, HS). Even though maximum oxytocin dose was high with high dose method, this was associated with significantly shorter times spent in the labor and delivery. In multigravdia high dose regimen associated slightly high maximum oxytocin dose compared to low dose regimen, however it was not significant. (Table 2)

\section{Discussion}

Complicated labour is considered by strangely sluggish labour development occurs from bungling uterine contractions, irregular fetal presentation or position, insufficient bony pelvis or deformity of the pelvic soft tissues of the mother. Data recommends that up to one third of first-time mothers experience hold-up in the first stage of labour. $^{4}$

Augmentation of labour has frequently been utilized to take care of deferred labour when uterine contractions are measured to be inadequately tough or improperly synchronized to dilate the cervix. Labour augmentation has usually been carry out with the utilization of intravenous oxytocin infusion and/or artificial rupture of amniotic membranes. ${ }^{7,8}$

On account of the findings of the study there was no major difference in deference to age, height, weight and gestational age when assessment was done between the two groups. The present study was in harmonization of Merrill et al. ${ }^{13}$

In our study it was seen that labour duration was significantly shortened in high dose groups. Shortening of both augmentation to delivery interval and second stage duration was seen. Our study could not be compared with most of the studies as these studies compare total labor duration and most of studies compare duration in hours. But all the studies show similar results of shortened labor duration with high dose regimen.

In another study done in primigravdia by Good bid et al., AD interval was significantly less in high dose than low dose. Even though AD interval was shorter in Bid good study, was longer than in our study. It may be because of shorter interval of waiting period for augmentation after functional dystocia is diagnosed in our study. ${ }^{14}$

In our study maximum oxytocin was significantly high in high dose group compared with low dose group. Our results are similar to that of most other studies by Merrill et al., Xenakis et al., Satin et al., Sadler et al. In our study same results of high maximum oxytocin dose in primigravida in high dose group was seen, but this difference was not seen with multigravida.

Mode of delivery did not differ in both groups in our study, without any increased spontaneous deliveries in high dose group. No significant increase in caesarean in low dose group was seen. These results were comparable with the results of Satin et al. Where as in study by Xenakis et al., low dose group was associated with high caesarean rate. It may because of use of very low dose $1 \mathrm{mU}$ of oxytocin for augmentation. 


\section{Conclusion}

In the present study it has been observed that high dose oxytocin clearly had several advantages, when used for ineffective spontaneous labor, following rupture of membrane sin primigravida. High dose labor augmentation in primigravdia was associated with shorter augmentation to delivery interval by $1 \mathrm{hr} 15 \mathrm{~min}$ and also moderate shortening of second stage of labor. Although labour augmentation with high dose in primigravida was associated with significantly more maximum oxytocin dose, it was associated with shorter labor duration without any apparent adverse maternal outcomes or fetal morbidity and mortality.

\section{Source of Funding}

None.

\section{Conflict of Interest}

The author(s) declare(s) that there is no conflict of interest.

\section{References}

1. Begum R. Labor Dystocia. In: Patient Management in Obstetrics \& Gynecology; 2018. p. 61

2. Romano AM, Lothian JA. Promoting, Protecting, and Supporting Normal Birth: A Look at the Evidence. J Obstet, Gynecol Neonatal Nurs. 2008;37(1):94-105. doi:10.1111/].1552-6909.2007.00210.x

3. Hodnett ED. Pain and women's satisfaction with the experience of childbirth: a systematic review. Am J Obstet Gynecol. 2002;186:16072 .

4. Neal JL, Ryan SL, Lowe NK, Schorn MN, Buxton M, Holley SL, et al. Labor Dystocia: Uses of Related Nomenclature. J Midwifery Women's Health. 2015;60(5):485-98. doi:10.1111/jmwh.12355.

5. Dawood MY. Pharmacologic stimulation of uterine contraction. Semin Perinatol. 1995;19(1):73-83. doi:10.1016/s0146-0005(95)80049-2
6. Hofmeyr GJ. Induction and augmentation of labour. In: Dewhurst's textbook of obstetrics and gynaecology. Oxford: Blackwell; 2007. p. $205-12$.

7. Organization WHO: WHO recommendations for augmentation of labour: World Health Organization; 2014.

8. Mander R. Caesarean: just another way of birth? Routledge; 2007.

9. Merrill DC, Zlatnik FJ. Randomized, double-masked comparison of oxytocin dosage in induction and augmentation of labor. Obstet Gynecol. 1999;94:455-63.

10. Hayes EJ, Weinstein L. Improving patient safety and uniformity of care by a standardized regimen for the use of oxytocin. Am J Obstet Gynecol. 2008;198(6):622.e1. 101:10.1016/1.ajog.2008.01.039.

11. Bugg GJ, Siddiqui F, Thornton JG. Oxytocin versus no treatment or delayed treatment for slow progress in the first stage of spontaneous labour. Cochrane Database Syst Rev. 2013; do1:10.1002/14651858.cd007123.pub3.

12. Phaneuf S, Linares BR, Raja RT, Mackenzie I, Bernal AL. A: Loss of myometrial oxytocin receptors during oxytocin-induced and oxytocinaugmented labour. J Reprod Fertil. 2000;120:91-8.

13. Ballard RA, Truog WE, Cnaan A, Martin RJ, Ballard PL, Merrill JD, et al. Inhaled Nitric Oxide in Preterm Infants Undergoing Mechanical Ventilation. $\quad N$ Engl J Med. 2006;355(4):343-53. do1:10.1056/nejmoa061088.

14. Conde-Agudelo A, Romero R, Kusanovic JP. Nifedipine in the management of preterm labor: a systematic review and metaanalysis. Am J Obstet Gynecol. 2011;204(2):134.e1. doi:10.1016/1.ajog.2010.11.038.

\section{Author biography}

Girija C Bellad, Associate Professor

Mahesweta Guru, Assistant Professor

Cite this article: Bellad GC, Guru M. Evaluation between low dose and high dose oxytocin for labour augmentation in fetal outcome. Indian $J$ Obstet Gynecol Res 2020;7(4):509-512. 